\title{
A COMPACT INTEGRATED HYPERSPECTRAL IMAGER MADE BY GRAY-TONE LITHOGRAPHY
}

\author{
M. Christophersen ${ }^{l *}$, A. J. Boudreau ${ }^{l}$, M. J. DePrenger ${ }^{2}$, and M. K. Yetzbacher ${ }^{l}$ \\ ${ }^{1}$ US Naval Research Laboratory, Washington, District of Columbia, USA \\ ${ }^{2}$ Tekla Research Corporation, Dumfries, Virginia, USA
}

\begin{abstract}
Optical elements of precise thickness profiles are desired for instruments such as coronagraphs [1], wavefront coding systems [2], and compact hyperspectral imagers [3, 4]. Lack of subwavelength precision in the fabrication of these optical elements has required additional optical components, reducing the manufacturing yield and degrading performance. Here, we demonstrate a fabrication technique allowing for the control of surface fabrication errors of less than $1 \mathrm{~nm}$ and use this technique to fabricate a compact hyperspectral imager with the capability of high yield manufacturing.
\end{abstract}

\section{INTRODUCTION}

Any micro-fabricated optical device is in some way limited by the "fabrication errors". Fabrication errors are deviations of the actual optical surface from the designed surface. These errors arise from imperfections in the fabrication process. Wavefronts produced by imperfect optical surfaces are also imperfect, suffering from aberrations. For optical elements of precise thickness profiles, primary concerns are the deviations in material thickness, homogeneity, and surface smoothness. A "classical optical device" with a defined thickness and a low roughness requirement is the Fabry-Pérot (FP) etalon. A FP etalon is typically made of a transparent plate with two reflecting surfaces. Its transmission spectrum as a function of the incident wavelength exhibits peaks of large transmission corresponding to resonances of the etalon cavity.

Multiple FP etalons can be combined and used as a hyperspectral spectrometer. Our device exploits the fact that the transmission through such a FP etalon exhibits sharp peaks at cavity resonances and is very small between those. Our Hyperspectral imagers divide the light spectrum into finely tuned interferograms by using multiple FP etalons. This enables the imager to capture the "spectral signature" of an object. Applications for hyperspectral imagers range from medical diagnosis to security applications. There are multiple ways to achieve hyperspectral imaging. Conventional spectrometers typically use dispersive elements uncoupled from the photosensitive detector to separate light into its spectral components, requiring space and precise alignment of optics. The requirement of holding imaging optics and dispersive optics in precise alignments adds substantially to the size, weight, and assembly costs of optical systems. The size, weight and cost of spectrometers can be reduced by building an integrated optical device on the image sensor.

Several types of integrated spectrometers have previously been demonstrated by using micro-scale FP etalon array. A microfabricated FP etalon is made of a transparent medium bounded by two reflecting surfaces to create an optical cavity. The transmission spectrum of the cavity exhibits peaks of transmission corresponding to resonances of the optical cavity. The microfabrication challenge for a FP etalon array is the formation of multiple, flat levels. The FP etalons described in [3, 4] use combinatorial etching. A straightforward implementation of the staircase structure would use successive lithographic patterning and etching steps. This technique requires a large number of processing steps in order to produce a staircase with $\mathrm{k}$ steps, with $\mathrm{k}$ typically being $>50$ or even 100 . By using a combinatorial patterning process the number of required steps can be reduced to $\log _{2}(k)$. Sabatyan et al. have used a single gray-tone patterning step to produce a ramp without discrete levels [6]. Our microfabrication process for the FP Etalon array is based on a single gray-tone lithographic step on a bi-layer stack, see microfabrication section for details.

\section{MICROFABRICATION}

Our approach allows fabrication with a single lithograph-etch step followed by a chemical etch step. The number of distinct levels achievable is independent from the number of lithograph steps and is independent of the number of resolvable gray-scale lithography levels. This allows for a processing sequence that is much faster and more robust than previously demonstrated.

Our process is based on gray-tone lithography on a bi-layer stack, see Figure (1) for a schematic of the basic process sequence. Standard lithography generates a "black-white" image with a distinct top-down, two-level, profile. Gray-tone lithography on the other hand can generate full three-dimensional (3-D) resist structures like ramps. The variability of photoresist exposure and development in gray-tone lithography can be partially mitigated to create smooth photoresist structures. The 3-D resist structure can be transferred by a RIE (reactive ion etching) step into almost any type of material. We transfer a gray-tone resist ramp into a bi-layer stack a few micrometers thickness. After the RIE step, a roughened surface remains due to variability in material, photoresist homogeneity, and the random nature of the etch process. This has historically limited the resolvable gray-tone levels. In our process, a chemical etch then digitizes the levels. Each bilayer is comprised of two dielectric materials wherein one material acts as a chemical etch stop (i.e. one etches quickly and the other one very slow when exposed to the same etch process). The etch stop of each bilayer defines these individual levels. Our fabrication sequence is based on four basic process steps, as shown in Figure (1).

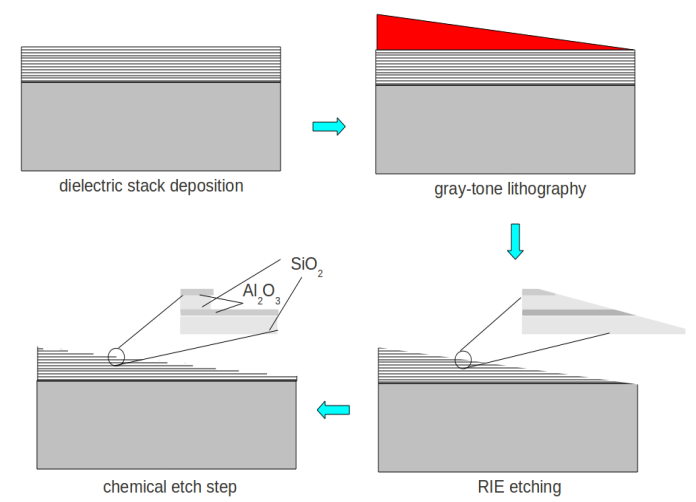

Figure 1: Basic process sequence, (i) bi-layer deposition of bilayer stack, (ii) gray-tone lithography, (iii) transfer of into stack by RIE, and (iv) chemical etch, formation of individual levels. 
Figure (2) shows schematically the finished etalon on a standard image sensor. Our device utilized a commercially available imaging sensor provided by Aptina ${ }^{\circledR}$ (MT9V111), a standard FSI (front side illuminated) CMOS imager. The top mirrors were always thin gold layers $(10 \mathrm{~nm}$ with $2 \mathrm{~nm}$ Ti adhesion layer). Two types of bottom mirrors were used: (i) $2 \mathrm{~nm} \mathrm{Ti} / 10 \mathrm{~nm}$ $\mathrm{Au}$ (like top mirror) or a (ii) dielectric stack of tantalum pentaoxide, $\mathrm{Ta}_{2} \mathrm{O}_{5}$, and $\mathrm{SiO}_{2}$. Our silicon test substrates had $\mathrm{Au}$ bottom mirrors, and the Aptina imager had the dielectric stack as bottom mirror.

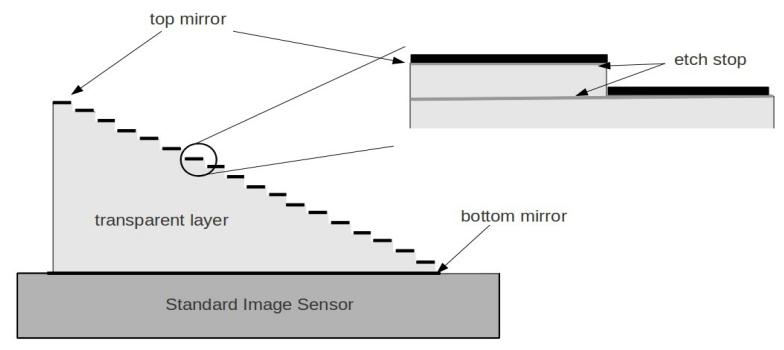

Figure 2: Schematic of the Etalon structure on a standard image sensor. The individual FP levels are defined by etch stops. Top and bottom mirrors form the optical cavity.

The dielectric stack can be deposited by different thin film deposition techniques. Plasma-enhanced chemical vapor deposition (PECVD) and atomic layer deposition (ALD) were chosen for our process. The stack is comprised of two materials (A and B). A is always deposited on top of $\mathrm{B}$; so the stack is comprised of multiple A-B bilayers. The refractive indices of material A and B need to similar in value to avoid unwanted reflections during the numerous interface changes. One material of the stack (e.g. B) will act as an "etch stop" (described below). Silicon dioxide $\left(\mathrm{SiO}_{2}\right)$ and alumina $\left(\mathrm{Al}_{2} \mathrm{O}_{3}\right)$ were used as components for the bi-layers. The number of etalon levels is determined by the number of bi-layers within the stack and the geometry of the gray-tone photoresist structure.

Gray-tone lithography is ideal for the fabrication of 3-D micro-structures. Gray-tone lithography is a specialized lithography process which results in continuously variable resist profiles. A gray-tone optical mask is used to transmit only a portion of the intensity of incident light, partially exposing sections of a positive photoresist to a certain depth. This exposure renders the top portion of the photoresist layer more soluble in a developer solution, while the bottom portion of the photoresist layer remains unchanged. The number of resolvable levels in gray-tone lithography has been limited by photoresist exposure nonlinearity, variability in development, and material homogeneity to less than 80 levels under common conditions. Continuous structures can be produced by heating the photoresist to smooth out surface nonuniformity.

The developed photoresist structures may be transferred, for example by plasma etching, to reproduce a scaled or unscaled version of the three dimensional structure in a target material. As the etch proceeds the photoresist mask slowly erodes exposing the underlying material, in our case a dielectric stack, to the high etch rate plasma. Gray-scale technology relies on specifically developed RIE chemistries and plasma conditions to control the relative etch rate of the dielectric stack to photoresist called "etch selectivity". This aspect defines the final vertical dimensions of a 3D structure. RIE etching is known to create surface nonuniformities due to the random nature of the etching process and material inhomogeneity, see Figure 3 for an optical micrograph.

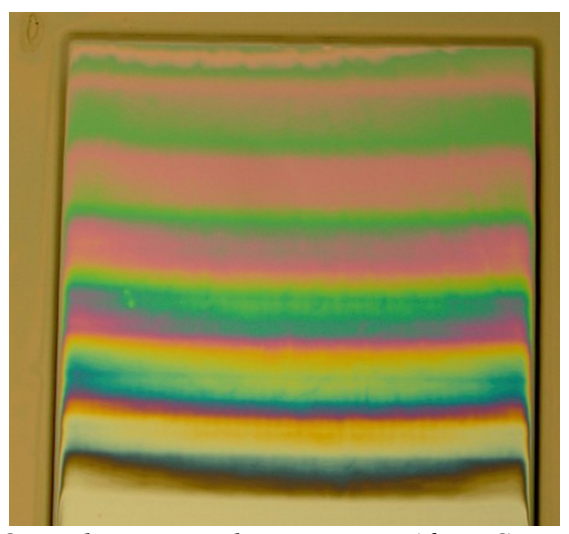

Figure 3: Optical micrograph, top-view: After ICP etching there are no distinct levels.

After transferring the gray-tone resist into the stack a chemical etch step is performed. The chemical selectively only etches one material of the bi-layer (e.g. when A is etched B is not). This selective chemical etch step will transform the ramp into distinct levels. Vapor hydrofluoric acid (HF) was used to etch $\mathrm{SiO}_{2}$ selectively over the $\mathrm{Al}_{2} \mathrm{O}_{3}$. This leads to digitally distinct levels at a resolution controlled by the deposition technique of the bilayer stack which in this case is atomic layer precision. Figure (4) shows the stair-step structure on a test substrate. Figure (5) shows an optical profiometer scan from 5 individual steps.

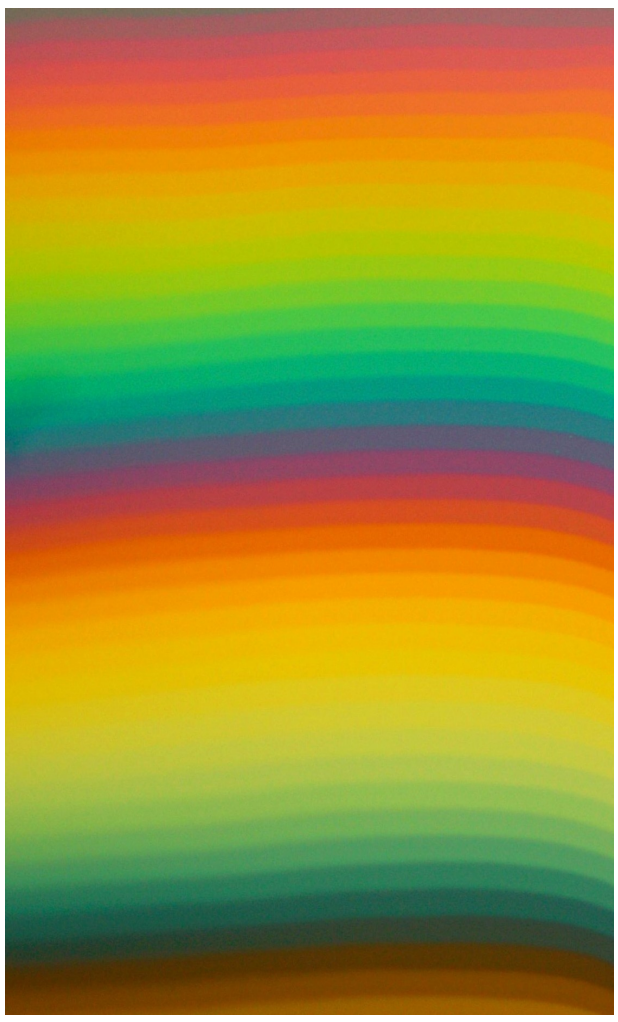

Figure 4: Optical micrograph after vapor HF etching, top view, white light fringe pattern (step width $\sim 10 \mu \mathrm{m}$, bi-layer height 10 nm: $\left.7 \mathrm{~nm} \mathrm{SiO} O_{2}+3 \mathrm{~nm} \mathrm{Al}_{2} \mathrm{O}_{3}\right)$. The different optical levels ( 70 distinct levels) are clearly visible. The sharp transitions between steps are due to a finely digitized staircase. This micrograph only shows a section of our ramp. Overall, the Fabry-Pérot etalon array is comprised of 140 distinct levels. 


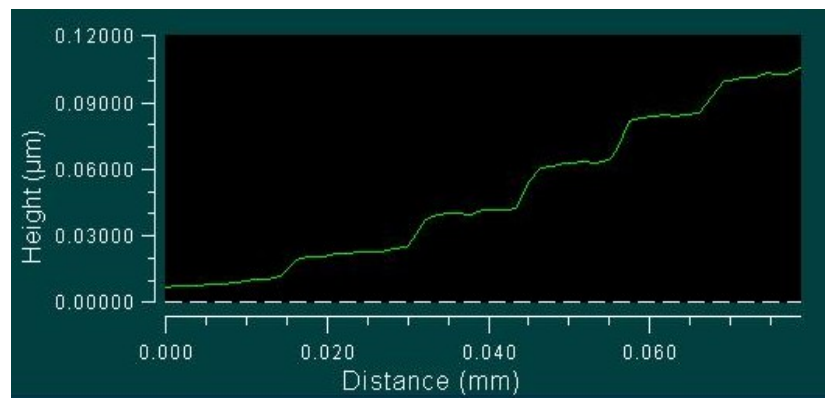

Figure 5: Optical profilometer scan from 5 individual steps. The roughness of each level is $\sim 1 \mathrm{~nm} \mathrm{rms.}$

\section{METHODS}

The gray-tone lithography was done by using a Novolak resist (AZ P4330 Clariant, $\sim 3 \mu \mathrm{m}$ thick). According to standard softbake procedure the wafer was heated to $100^{\circ} \mathrm{C}$ for $60 \mathrm{~s}$ on a hotplate in order to drive solvents out of the resist prior to UV exposure. We used "diffuser-based" gray-tone lithography, see [7] for details, with a 15 second exposure time. According to standard development procedure the resist was developed using the puddle method. 1:4 DI water to $400 \mathrm{~K} \mathrm{AZ} \mathrm{developer} \mathrm{was} \mathrm{used} \mathrm{for} \sim 40 \mathrm{sec}$ which was followed by a sound DI (de-ionized) water rise. After development the resist was hard-baked at $150^{\circ} \mathrm{C}$ for 120 seconds. The hard-bake makes the resist more resistant during the RIE etching step. Figure (4) shows an optical micrograph from a graytone resist ramp made by "diffuser gray-tone lithography". The numerous distinct etalon heights or clearly shown.

The gray-tone resist patterns were transferred into the stack via an ICP (induction coupled plasma) RIE etch process step. The ICP etch was performed using an Oxford 100 fluorine etcher (30 sccm $\mathrm{CF}_{4}, 20$ mTorr, $20 \mathrm{~W} \mathrm{FW}$ power, $500 \mathrm{~W}$ ICP power, 90 min etch time).

We used vapor HF (hydrofluoric acid) for the chemical etch step (Primaxx Etch System). Vapor HF only etches the silicon oxide and leaves the alumina intact. Hydrofluoric acid's reaction with $\mathrm{SiO}_{2}$ forms water acting as a catalyst which will cause aqueous HF to etch the alumina. Commercial HF vapor etch systems minimize the formation of $\mathrm{HF}$ (aqu.) by injecting alcohol into the system.

\section{RESULTS AND DISCUSSION}

Our substrate was a standard imaging sensor, MT9V111 from Aptina ${ }^{\circledR}$. Figure (6) displays the spectral response from the FP Etalons with $0.64,1.0$, and $1.5 \mu \mathrm{m}$ effective heights on an imager. The light detection was done with the Aptina imager array underneath the etalon structure. The transmission versus frequency for FP Etalons is normally described with an Airy function. Figure (7) shows the calculated (Airy Function) FP etalon level heights derived from measurement of the spectral sensitivity of our device using a sequence of images captured under monochromatic light. The fabricated device achieves $10 \mathrm{~nm}$ discrete level resolution, enabling compact, hyperspectral imaging with high-yield device manufacture. Figure (8) shows a photograph from a prototype device.

Figure (9) shows a SEM, scanning electron microscope, cross-sectional micrograph from a thick Etalon. The CMOS chip with top wiring and its passivation is visible. The bottom mirror is comprised of $\mathrm{Ta}_{2} \mathrm{O}_{5}$, and $\mathrm{SiO}_{2}$ layers. One cannot distinguish individual $\mathrm{SiO}_{2}$ and $\mathrm{Al}_{2} \mathrm{O}_{3}$ bi-layers.

Since the MT9V111 is a FSI CMOS imager, normally microlenses are placed on top of the passivation layer. These

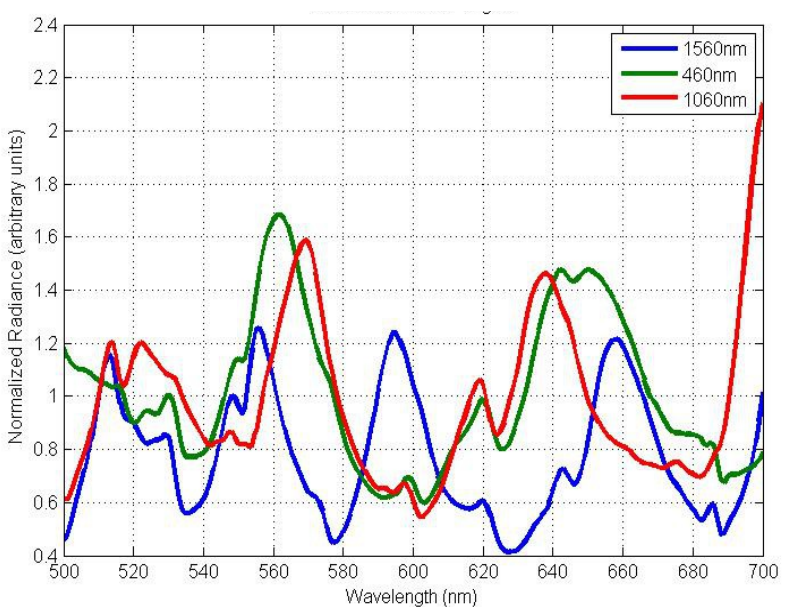

Figure 6: Fringes from Fabry-Pérot Etalons 0.64, 1.0, and $1.5 \mu \mathrm{m}$ effective heights. The light detection was done with a MT9V111 from Aptina.

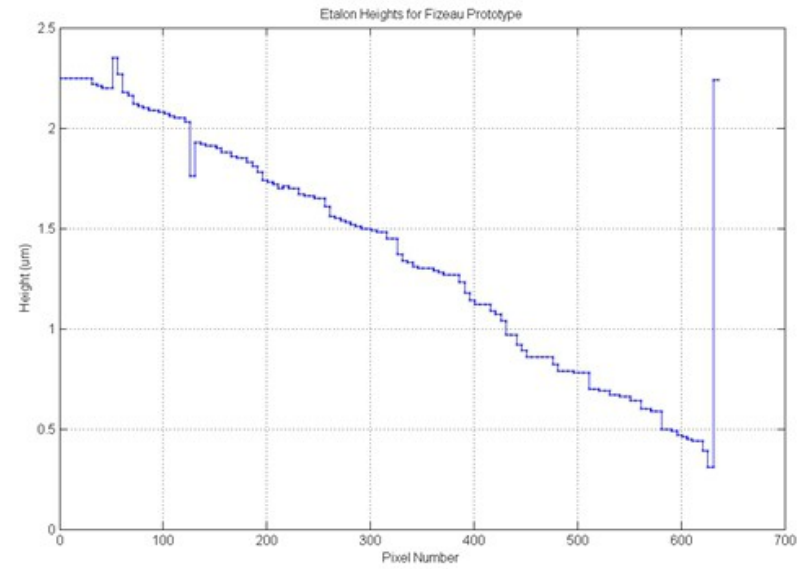

Figure 7: Plot of distinct levels calculated from illumination of an Aptina imager with monochromatic light using a superposition Airy function.

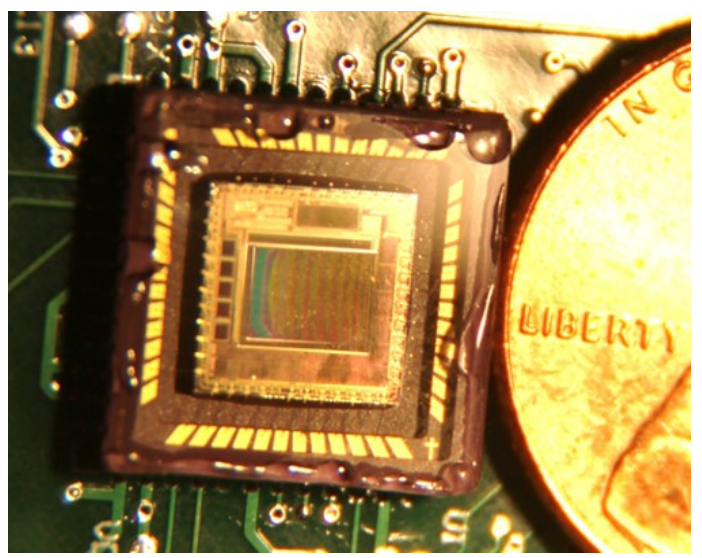

Figure 8: Photograph of the prototype device with a penny for scale. The wedged etalon array on the sensing chip is composed of 140 distinct levels. The striping of the first two layers is clearly visible on this scale. Since the color resolution of our device is much finer than that of the camera used to take this image, groups of etalon levels may appear to be the same color. A moiré pattern of interfering colors is visible over the larger steps. The shortest steps have etalon thicknesses of approximately $150 \mathrm{~nm}$, while the tallest steps have etalon thicknesses of approximately 1.5 microns. 


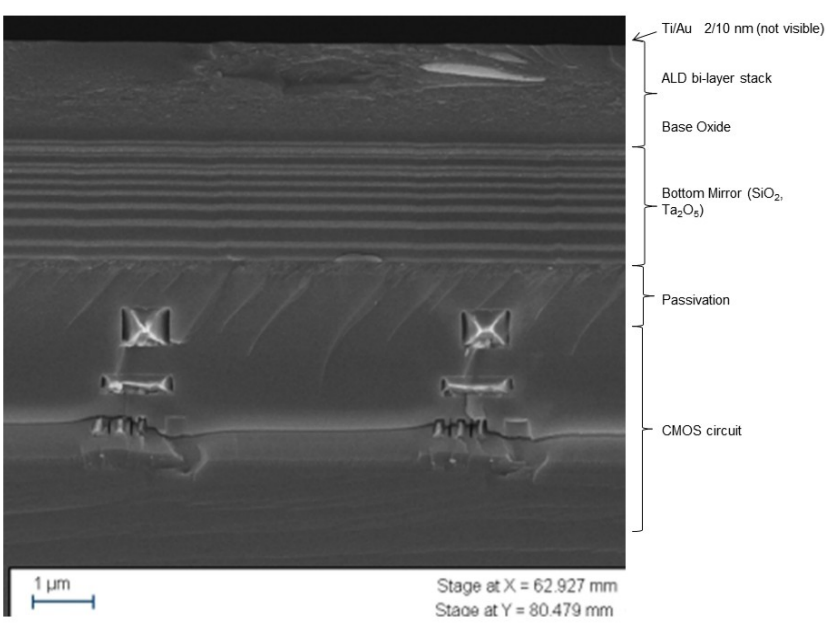

Figure 9: SEM micrograph, cross-section from the final prototype device. This image shows a thick Etalon; most of the bi-layer stack is visible.

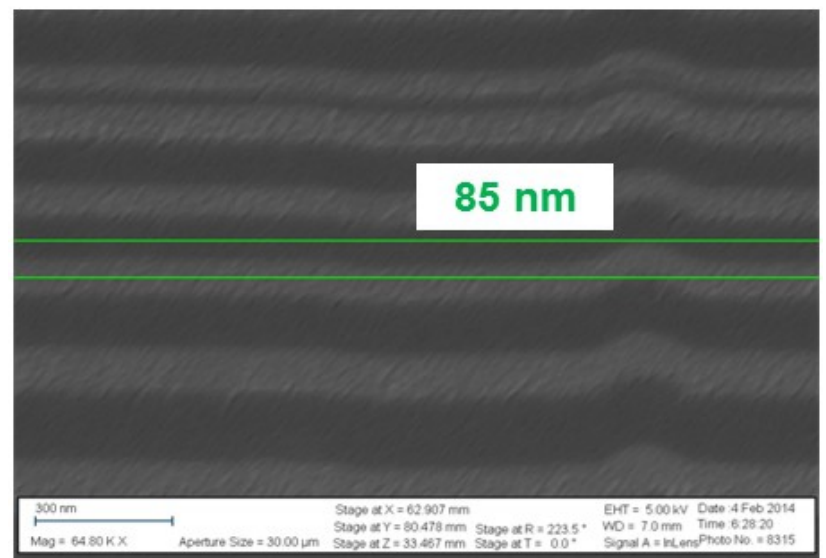

Figure 10: SEM micrograph, cross-section, bottom mirror stack. The interconnect lines introduce surface steps.

lenses are engineered to gather the light and confine it to a narrowed beam as it passes through the stack of interconnect metals and isolation. Our current design does not includes any lenses, so there is light scattering from the stack of interconnect metals, introducing noise and acting as parasitic mirrors. Furthermore, the interconnect lines introduce some small steps on the imager top surface, see Figure (10). Since all of our coating techniques are conformal these steps will remain and introduce noise into the system.

\section{FUTURE WORK}

Future work will include improving the finesse of the Etalon. The finesse describes the narrowness of the peaks relative to the spacing between the peaks.

Since the MT9V111 is a FSI CMOS imager, normally microlenses are placed on top of the passivation layer. These lenses are engineered to gather the light and confine it to a narrowed beam as it passes through the stack of interconnect metals and isolation. Our current design does not include any lenses, leading to light scattering and parasitic mirrors. Furthermore, the interconnect lines introduce some small steps on the imager top surface, see Figure (10). Since all of our coating techniques are conformal these steps will remain and introduce noise into the system.

In the future, we will switch to BSI, back side illuminated, CMOS imagers as substrates for our FP Etalons in order to circumvent the problems with respect to the interconnect lines. A pixel built for backside illumination eliminates the need to pass light through the layers of metal interconnections. Hence, no light scattering can occur on interconnects. Since BSI CMOS imagers are made by thinning down and bonding wafers, the top surface is extremely flat. This flatness will lead to better FP Etalons on BSI CMOS imagers. Furthermore, compared to FSI sensor, BSI senor shows much better quantum efficiency and chief ray angle.

\section{SUMMARY}

We introduce a new fabrication technique that allows for the creation of an arbitrary number of distinct levels with a single gray-scale lithography technique. Our technique allows the fabrication of distinct levels of $1 \mathrm{~nm}$ rms flatness with $10 \mathrm{~nm}$ step heights. In this paper, we present the fabrication technique and characteristics of an active imager.

\section{ACKNOWLEDGMENTS}

This work was funded by the Office of Naval Research (ONR). We thank Aptina for helpful discussion with respect to the CMOS imager. Furthermore, we would like to thank the Institute for Nanoscience (NSI) at the U.S. Naval Research Laboratory and the NSI staff members - "thanks Dave".

\section{REFERENCES}

[1] J. Mazoyer, P. Baudoz, R. Galicher, M. Mas and G. Rousset, "Estimation and correction of wavefront aberrations using the self-coherent camera: laboratory results", Astronomy \& Astrophysics, 557, A9 (2013).

[2] C.-C. Chang and C.-C. Lee., "Effect of surface manufacturing error of cubic phase mask in wavefront coding system", Optics Express, 17, 6245 (2009).

[3] N. Tack, A. Lambrechts, P. Soussan, and L. Haspeslagh, "A compact, high-speed, and low-cost hyperspectral imager", Proceedings of Silicon Photonics VII, San Francisco, CA, 01/21/12-01/26/12, SPIE, Bellingham (2012), pp. 8266-8279.,

[4] S.-W. Wang, C. Xia, X. Chen, W. Lu, M. Li, H. Wang, W. Zheng, and T. Zhang, "Concept of a high-resolution miniature spectrometer using an integrated filter array", Optics Letters, 32, 632 (2007).

[5] A. Emadi, H. Wu, S. Grabarnik, G. De Graaf, K. Hedsten, P. Enoksson, J.H. Correia, and R.F. Wolffenbuttel, "Fabrication and characterization of IC-Compatible Linear Variable Optical Filters with application in a micro-spectrometer", Sensor and Acutators A, 162, 400 (2010).

[6] A. Sabatyan and A. Hemmat, "Study of a novel and simple method of generating binary mask for microprism fabrication", Applied Optics, 51, 525 (2012).

[7] M. Christophersen and P. F. Phlips, "Gray-tone lithography using an optical diffuser and a contact aligner", Applied Physics Letters, 92, 194102 (2008).

\section{CONTACT}

*Marc Christophersen, tel: +1-202-404-2884 marc.christophersen@nrl.navy.mil 